\title{
Preparing a Mechanical Oscillator in Non-Gaussian Quantum States
}

\author{
Farid Khalili, ${ }^{1}$ Stefan Danilishin, ${ }^{1}$ Haixing Miao, ${ }^{2}$ Helge Müller-Ebhardt, ${ }^{3}$ Huan Yang, ${ }^{4}$ and Yanbei Chen ${ }^{4}$ \\ ${ }^{1}$ Physics Faculty, Moscow State University, Moscow 119991, Russia \\ ${ }^{2}$ School of Physics, University of Western Australia, WA 6009, Australia \\ ${ }^{3}$ Max-Planck Institut für Gravitationsphysik (Albert-Einstein-Institut) and Leibniz. Universität Hannover, \\ Callinstr. 38, 30167 Hannover, Germany \\ ${ }^{4}$ Theoretical Astrophysics 130-33, California Institute of Technology, Pasadena, California 91125, USA \\ (Received 5 February 2010; revised manuscript received 19 April 2010; published 11 August 2010)
}

\begin{abstract}
We propose a protocol for coherently transferring non-Gaussian quantum states from an optical field to a mechanical oscillator. We demonstrate its experimental feasibility in future gravitational-wave detectors and tabletop optomechanical devices. This work not only outlines a feasible way to investigate nonclassicality in macroscopic optomechanical systems, but also presents a new and elegant approach for solving non-Markovian open quantum dynamics in general linear systems.
\end{abstract}

DOI: 10.1103/PhysRevLett.105.070403

Introduction.-Recently, the attention of broad scientific communities has been drawn to the investigation of the quantum behavior of macroscopic mechanical oscillators in optomechanical devices [1]. These activities are motivated by (i) the necessity to achieve and go beyond the Standard Quantum Limit for high-precision measurements with mechanical probes [2], (ii) the test and interpretation of quantum theory with macroscopic degrees of freedom [3], and (iii) quantum information processing with optomechanical devices [1]. Non-Gaussian quantum states, such as Fock states, lie at the heart of all these endeavors $[4,5]$. A $6 \mathrm{GHz}$ micromechanical oscillator has been lately prepared in a single-quantum state, by coupling it to a superconducting qubit [6]. This significant achievement is attributable to the intrinsic nonlinearity in the oscillator-qubit system $[7,8]$. For optomechanical systems to achieve nonlinearity in the quantum regime, the zeropoint displacement $x_{q}=\left(\frac{\hbar}{2 m \omega_{m}}\right)^{1 / 2}$ of the oscillator needs to be comparable to the cavity linear dynamical range - the ratio of the optical wavelength $\lambda$ to the cavity finesse $\mathcal{F}$ and this condition reads [9-12]

$$
\lambda /\left(\mathcal{F} x_{q}\right) \lesssim 1
$$

Since, in typical experiments, $\lambda=10^{-6} \mathrm{~m}$ and $\mathcal{F} \leq 10^{6}$, this requires $x_{q} \geq 10^{-12} \mathrm{~m}$, which is rather challenging to achieve. For large-scale optomechanical devices, e.g., an interferometric gravitational-wave detector with $m=$ $40 \mathrm{~kg}$ and $\omega_{m} / 2 \pi=1 \mathrm{~Hz}$ [13], $x_{q} \sim 10^{-18} \mathrm{~m}$; on the small scale, e.g., the one reported in Ref. [10], $m=$ $10 \mathrm{ng}$ and $\omega_{m} / 2 \pi=10^{5} \mathrm{~Hz}$, which gives $x_{q} \sim 10^{-15} \mathrm{~m}$. Both are far away from the nonlinear regime.

In this Letter, we propose a protocol for preparations of non-Gaussian states with optomechanical devices, which does not require optomechanical nonlinearity. The idea is to inject non-Gaussian optical states, e.g., a single-photon pulse created by a cavity QED process [14-16], into the dark port of interferometric optomechanical devices (cf. Fig. 1). The radiation-pressure force of the single-
PACS numbers: 03.65.Ta, 03.65.Ud, 04.80.Nn, 42.50.Dv

photon pulse on the oscillator is coherently amplified by the classical pumping in the bright port, and the nonGaussian states can be efficiently transferred from the optical field to the oscillator. The qualitative condition for such a state transfer, as will be shown, is

$$
\lambda /\left(\mathcal{F} x_{q}\right) \lesssim \sqrt{N_{\gamma}} .
$$

Here, $N_{\gamma}=I_{0} \tau /\left(\hbar \omega_{0}\right)$, with $I_{0}$ the laser power into the bright port and $\omega_{0}$ the frequency, is the number of pumping photons entering the bright port, within the single-photon pulse duration $\tau$. We gain a significant factor of $\sqrt{N_{\gamma}}$ which makes this protocol experimentally feasible.

Transferring quantum states from an optical field to a mechanical oscillator with other protocols has been discussed by many pioneering works [17-20]. To motivate experiments with this protocol, it is crucial to understand (i) the dynamics: how the mechanical oscillator interacts with the photon pulse, and (ii) the conditional process: how the continuous measurement affects the final quantum state of the oscillator. For the dynamics, we will study the full quantum dynamics without using either the rotating-wave approximation [17,18], or the three-mode approach $[19,20]$, because the interaction can be shorter than one
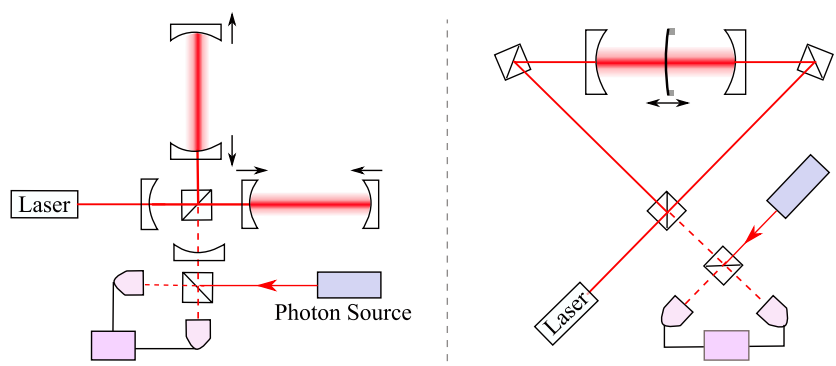

FIG. 1 (color online). Possible experimental schemes: (i) an interferometric gravitational-wave detector with $\mathrm{kg}$-scale test masses (left) [13], and (ii) a tabletop coupled cavity scheme with a ng-scale membrane (right) [10]. 
mechanical oscillation period to minimize the thermal decoherence effect-a strong optomechanical coupling. For the conditional process, nontrivial quantum correlations at different times in the photon pulse make the open quantum dynamics highly non-Markovian, which does not allow a transparent study with the standard Stochastic Master Equation (SME) approach [21-25]. We develop a path-integral approach. It solves the non-Markovian dynamics elegantly, and gives an explicit expression for the final state of the mechanical oscillator, which is valid for general linear dynamics.

Order-of-magnitude estimate.-The model of interferometric optomechanical devices is shown schematically in Fig. 2. The oscillator position $\hat{x}$ is coupled to a thermal bath and also the cavity mode $\hat{a}$ (mediated by radiation pressure), which in turn interacts with the ingoing $\hat{a}_{\text {in }}$ and outgoing $\hat{a}_{\text {out }}$ optical fields. To gain a qualitative picture, we first make an order-of-magnitude estimate by considering a simple case in which the cavity bandwidth is large, with $\hat{a}$ adiabatically eliminated, and the oscillator is a free mass (frequency $\omega_{m} \sim 0$ ), with the thermal force ignored. The Heisenberg equations of motion read

$$
\begin{gathered}
\dot{\hat{x}}(t)=\hat{p}(t) / m, \quad \dot{\hat{p}}(t)=\alpha \hat{a}_{1}(t), \\
\hat{b}_{1}(t)=\hat{a}_{1}(t), \quad \hat{b}_{2}(t)=\hat{a}_{2}(t)+(\alpha / \hbar) \hat{x}(t) .
\end{gathered}
$$

Here, the coupling constant $\alpha \equiv 8 \sqrt{2}(\mathcal{F} / \lambda) \sqrt{\hbar I_{0} / \omega_{0}} ; \hat{a}_{1,2}$ $\left(\hat{b}_{1,2}\right)$ are the amplitude and phase quadratures of the ingoing field $\hat{a}_{\text {in }}$ (outgoing field $\left.\hat{a}_{\text {out }}\right)$ with $\hat{a}_{1} \equiv\left(\hat{a}_{\text {in }}+\right.$ $\left.\hat{a}_{\text {in }}^{\dagger}\right) / \sqrt{2}$ and $\hat{a}_{2} \equiv\left(\hat{a}_{\text {in }}-\hat{a}_{\text {in }}^{\dagger}\right) /(i \sqrt{2})$ (and the same for the relation between $\hat{b}_{1,2}$ and $\hat{a}_{\text {out }}$ ).

Suppose, at $t=-\tau$, the oscillator is prepared in some initial quantum state $\left|\psi_{m}\right\rangle=\int_{-\infty}^{\infty} \psi_{m}(x)|x\rangle d x$, and is interacting with a single photon up to $t=0$. With a short photon pulse, i.e., a short interaction duration, the oscillator position almost does not change, and we obtain

$$
\begin{gathered}
\hat{X}(0)=\hat{X}(-\tau), \quad \hat{P}(0)=\hat{P}(-\tau)+\kappa \hat{A}_{1}, \\
\hat{B}_{1}=\hat{A}_{1}, \quad \hat{B}_{2}=\hat{A}_{2}+\kappa \hat{X}(0) .
\end{gathered}
$$

The oscillator position and momentum are normalized: $\hat{X} \equiv \hat{x} / x_{q}$ and $\hat{P} \equiv \hat{p} / p_{q} ; \hat{A}_{j} \equiv \sqrt{\frac{2}{\tau}} \int_{-\tau}^{0} d t \hat{a}_{j}(t)(j=1,2)$

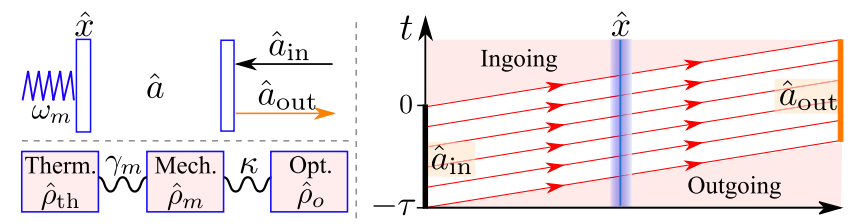

FIG. 2 (color online). A model of the optomechanical devices in Fig. 1 (upper left), its spacetime diagram (right), and the couplings (lower left). Ingoing and outgoing rays (tilted lines) are placed on the opposite sides of the oscillator world line (vertical) for clarity. The ingoing field contains a photon pulse, while the outgoing field-containing the information of the oscillator motion-is measured continuously. with uncertainty $\Delta \hat{A}_{j}=\left\langle\psi\left|\left(\hat{A}_{j}-\bar{A}_{j}\right)^{2}\right| \psi\right\rangle^{1 / 2}=1 ; \hat{B}_{j} \equiv$ $\sqrt{\frac{2}{\tau}} \int_{-\tau}^{0} d t \hat{b}_{j}(t) ; \kappa \equiv \alpha \sqrt{2 \tau} x_{q} / \hbar=16 \sqrt{N_{\gamma}} \mathcal{F} x_{q} / \lambda$. These two equations correspond to an evolution operator: $\hat{U}=$ $\exp \left[i \kappa \hat{A}_{1} \hat{X}\right]$ in the Schrödinger picture. The quantum state of the system at $t=0$ is simply $|\psi\rangle=\hat{U}\left|\psi_{o}\right\rangle\left|\psi_{m}\right\rangle$, where $\left|\psi_{o}\right\rangle$ is the initial optical state. Given a measurement of $\hat{B}_{2}$ with a precise result $y$, the oscillator is projected into a conditional quantum state: $\left|\psi_{m}^{c}\right\rangle=\left\langle y|\hat{U}| \psi_{o}\right\rangle\left|\psi_{m}\right\rangle$, which, in the coordinate representation $\psi_{m}^{c}(X) \equiv\left\langle X \mid \psi_{m}^{c}\right\rangle$, reads

$$
\psi_{m}^{c}(X)=\psi_{o}(y-\kappa X) \psi_{m}(X) .
$$

The optical state is mapped onto the mechanical oscillator. A significant mapping requires that $\psi_{o}(y-\kappa X)$ dominate over $\psi_{m}(X)$ in determining the profile of $\psi_{m}^{c}(X)$, or equivalently, the momentum uncertainty induced by the optomechanical interaction be larger than that from the initial state $\psi_{m}(X)$, i.e., $\kappa \Delta \hat{A}_{1}>\Delta \hat{P}(-\tau)$. Suppose the oscillator is initially in its ground state; we have $\Delta \hat{P}(-\tau)=1$. Since $\Delta \hat{A}_{1}=1$, this condition reads $\kappa>1$, i.e., $\lambda /\left(\mathcal{F} x_{q}\right)<$ $16 \sqrt{N_{\gamma}}$, which justifies Eq. (2).

In the above considerations, we have ignored the important thermal decoherence effect. In a real experiment, it is essential that the momentum fluctuations due to the thermal force within the interaction duration $\tau-$ $\Delta \hat{P}_{\text {th }}=\left(S_{F F}^{\text {th }} \tau\right)^{1 / 2} / p_{q}$ - should be small compared with that from the optomechanical interaction, namely, $\Delta \hat{P}_{\text {th }}<$ $\kappa$. In the high temperature limit, the force spectrum $S_{F F}^{\text {th }}$ of the thermal force is $2 m \omega_{m} k_{B} T / Q_{m}$, with $T$ the environmental temperature and $Q_{m}$ the mechanical quality factor. More explicitly, such a requirement is

$$
\lambda /\left(\mathcal{F} x_{q}\right) \sqrt{n_{\mathrm{th}} / Q_{m}} \sqrt{\omega_{m} \tau}<8 \sqrt{N_{\gamma}},
$$

with $n_{\text {th }} \equiv k_{B} T /\left(\hbar \omega_{m}\right)$ the thermal occupation number. These two conditions [cf. Eqs. (2) and (8)] set the benchmarks for a successful non-Gaussian state-preparation experiment. They can be satisfied with experimentally feasible specifications shown in Table I. The first row is close to that of a gravitational-wave detector, and the second row is close to that of a tabletop device in Ref. [10], with $\lambda=1 \mu \mathrm{m}$ and $\mathcal{F} \approx 10^{4}$ for both cases.

General formalism.- -Now, we start a quantitative study of the open quantum dynamics by first dividing the entire process from $t=-\tau$ to $t=0$ into $N$ segments, and later taking the continuous limit. The $n$-th segment consists of: (i) a free evolution, described by an evolution operator: $\hat{U}_{n} \equiv \exp \left[-i \hat{H}_{n} \tau /(N \hbar)\right]$ with $\hat{H}_{n}$ the system Hamiltonian at $t=(n-N) \tau / N$, and (ii) a measurement of the outgoing

TABLE I. Possible experimental specifications.

\begin{tabular}{lcccccc}
\hline \hline parameters & $m$ & $\frac{\omega_{m}}{2 \pi}$ & $Q_{m}$ & $T$ & $I_{0}$ & $\tau$ \\
\hline large scale & $40 \mathrm{~kg}$ & $1 \mathrm{~Hz}$ & $10^{8}$ & $300 \mathrm{~K}$ & $200 \mathrm{~W}$ & $1 \mathrm{~ms}$ \\
small scale & $10 \mathrm{ng}$ & $10^{5} \mathrm{~Hz}$ & $10^{7}$ & $4 \mathrm{~K}$ & $0.2 \mu \mathrm{W}$ & $0.02 \mathrm{~ms}$ \\
\hline \hline
\end{tabular}


field at a certain quadrature $\hat{y}=\hat{b}_{1} \cos \theta+\hat{b}_{2} \sin \theta$, described by a projection operator: $\hat{\mathcal{P}}_{n}=\delta\left(\hat{y}-y_{n}\right)$ with $y_{n}$ the measurement result of $\hat{y}$. After the entire process and conditioning on the measurement results $\boldsymbol{y}=\left(y_{1}, \cdots, y_{N}\right)$, the system is projected into a conditional quantum state,

$$
\hat{\rho}^{c}(\boldsymbol{y})=\hat{\mathcal{P}}_{y} \hat{\rho}_{i} \hat{\mathcal{P}}_{y}^{\dagger} / w(\boldsymbol{y}),
$$

with $\hat{\mathcal{P}}_{\boldsymbol{y}} \equiv \hat{\mathcal{P}}_{N} \hat{\mathcal{U}}_{N} \cdots \hat{\mathcal{P}}_{1} \hat{\mathcal{U}}_{1}$ and $w[\boldsymbol{y}] \equiv \operatorname{Tr}\left[\hat{\mathcal{P}}_{y} \hat{\rho}_{i} \hat{\mathcal{P}}_{y}^{\dagger}\right]$ the probability for obtaining measurement results $\boldsymbol{y}$. The initial density matrix $\hat{\rho}_{i}$ of the system is $\hat{\rho}_{\text {th }}(-\tau) \otimes$ $\hat{\rho}_{o}(-\tau) \otimes \hat{\rho}_{m}(-\tau)$, with $\hat{\rho}_{\text {th }}, \hat{\rho}_{o}$, and $\hat{\rho}_{m}$ for the thermal bath, optical field, and mechanical oscillator, respectively.

The conditional quantum state of the mechanical oscillator $\hat{\rho}_{m}^{c}$ is obtained by tracing out the degrees of freedom of both the thermal heat bath and the optical field, i.e.,

$$
\hat{\boldsymbol{\rho}}_{m}^{c}(\boldsymbol{y})=\operatorname{Tr}_{\mathrm{th}, o}\left[\hat{\boldsymbol{\rho}}^{c}(\boldsymbol{y})\right]=\operatorname{Tr}_{\mathrm{th}, o}\left[\hat{\mathcal{P}}_{\boldsymbol{y}} \hat{\boldsymbol{\rho}}_{i} \hat{\mathcal{P}}_{\boldsymbol{y}}^{\dagger}\right] / w(\boldsymbol{y}) .
$$

In the standard SME approach, such a trace operation is made right after each segment, which requires these degrees of freedom at different segments to be uncorrelated. However, this is not satisfied here due to quantum correlations among different segments (non-Markovian) arising from the nontrivial initial optical state. We apply a different approach based upon path integral. By using the facts that $\hat{\mathcal{P}}_{y}=\hat{\mathcal{U}}(\tau) \hat{\mathcal{P}}_{N}^{H} \hat{\mathcal{P}}_{N-1}^{H} \cdots \hat{\mathcal{P}}_{1}^{H} \equiv \hat{\mathcal{U}}(\tau) \hat{\mathcal{P}}_{y}^{H}$, where $\hat{\mathcal{U}}(\tau) \equiv$ $\prod_{n=1}^{N} \hat{U}_{n}$ (time-ordered) and $\hat{\mathcal{P}}_{n}^{H} \equiv \delta\left(\hat{y}_{n}-y_{n}\right)$ with $\hat{y}_{n} \equiv$ $\hat{U}^{\dagger}\left(\frac{n \tau}{N}\right) \hat{y} \hat{U}\left(\frac{n \tau}{N}\right)$, and the optical quadrature at different times commute: $\left[\hat{y}_{n}, \hat{y}_{n^{\prime}}\right]=0$, we obtain $\hat{\mathcal{P}}_{y}^{H}=$ $\prod_{n=1}^{N} \delta\left(\hat{y}_{n}-y_{n}\right)=\int \frac{d^{N} \xi}{(2 \pi)^{N}} \exp \left[i \sum_{n=1}^{N} \xi_{n}\left(\hat{y}_{n}-y_{n}\right)\right] . \quad$ In the continuous limit $N \rightarrow \infty$, the total projection operator can be rewritten as a path integral,

$$
\hat{\mathcal{P}}_{y}=\hat{U}(\tau) \int \mathcal{D}[\xi] \exp \left\{i \int_{-\tau}^{0} d t \xi(t)[\hat{y}(t)-y(t)]\right\},
$$

which allows us to take the entire measurement history and to trace out the optical field in a single step, instead of sequentially as in the SME approach.

To obtain an explicit expression for the conditional quantum state of the mechanical oscillator, i.e., its Wigner function, we evaluate the generating function,

$$
\mathcal{J}\left[\alpha_{x}, \alpha_{p} ; \boldsymbol{y}\right]=\operatorname{Tr}_{m}\left[e^{i \alpha_{x} \hat{x}+i \alpha_{p} \hat{p}} \hat{\boldsymbol{\rho}}_{m}^{c}(\boldsymbol{y})\right],
$$

which is related to the Wigner function by $W[x, p ; \boldsymbol{y}]=$ $\int \frac{d^{2} \boldsymbol{\alpha}}{(2 \pi)^{2}} e^{-i\left(\alpha_{x} x+\alpha_{p} p\right)} \mathcal{J}\left[\alpha_{x}, \alpha_{p} ; \boldsymbol{y}\right]$. From the facts that $\hat{U}(\tau)^{\dagger} \hat{x} \hat{U}(\tau)=\hat{x}(0)$ and $[\hat{x}(0), \hat{y}(t)]=0 \quad(t<0)$ (also true for $\hat{p}$ ), and the property $\hat{\mathcal{P}}_{\boldsymbol{y}}^{\dagger} \hat{\mathcal{P}}_{\boldsymbol{y}}=\hat{\mathcal{P}}_{\boldsymbol{y}}$, we obtain

$$
\mathcal{J}[\boldsymbol{\alpha} ; \boldsymbol{y}]=\operatorname{Tr}\left[e^{i \boldsymbol{\alpha} x_{0}^{\prime}} \hat{\mathcal{P}}_{y}^{H} \hat{\rho}_{i}\right] / w(\boldsymbol{y}),
$$

where vectors $\boldsymbol{\alpha} \equiv\left(\alpha_{x}, \alpha_{p}\right), \hat{\boldsymbol{x}}_{0} \equiv(\hat{x}(0), \hat{p}(0))$, and the superscript ' denotes the transpose. To proceed, we need to specify the initial density matrix $\hat{\rho}_{i}$ of the system. For the thermal bath in thermal equilibrium at temperature $T$, $\hat{\rho}_{\text {th }}(-\tau)=e^{-\hat{H}_{\mathrm{th}} / k_{B} T} / \operatorname{Tr}\left[e^{-\hat{H}_{\mathrm{th}} / k_{B} T}\right]$. For the optical field, we consider an arbitrary spatial profile $f(x / c)$ for the photon pulse of which the creation operator is $\hat{\Gamma}^{\dagger} \equiv$ $\int_{-\tau}^{0} d t f^{*}(t) \hat{a}_{\text {in }}^{\dagger}(t)$. In the $P$-presentation, a general initial state of such a mode can be written as $\hat{\rho}_{o}(-\tau)=$ $\int d^{2} \zeta P(\zeta)|\zeta\rangle\langle\zeta|$, with the vector $\zeta \equiv(\Re[\zeta], \Im[\zeta])$ and $|\zeta\rangle \equiv \exp \left[\zeta \hat{\Gamma}^{\dagger}-\zeta^{*} \hat{\Gamma}\right]|0\rangle$. Since the photon profile $f(t)$ will automatically set the interaction duration, we can extend $-\tau$ to $-\infty$, which is equivalent to adiabatically turning on the optomechanical interaction. Correspondingly, the initial-state information of the oscillator decays away before the optomechanical interaction starts, and thus does not influence $\hat{\rho}_{m}^{c}$.

Substituting in the initial state and Baker-CampbellHausdorff formula, the generating function becomes

$$
\mathcal{J}=\frac{1}{w(\boldsymbol{y})} \int d^{2} \zeta \mathcal{D}[\xi] e^{i\left[\xi^{*} \hat{\Gamma}-\zeta \hat{\Gamma}^{\dagger}, \hat{B}\right]}\left\langle 0\left|e^{i \hat{B}}\right| 0\right\rangle P(\boldsymbol{\zeta}),
$$

where $\hat{B} \equiv \boldsymbol{\alpha} \hat{\boldsymbol{x}}_{0}^{\prime}+\int_{-\infty}^{0} d t \xi(t)[\hat{y}(t)-y(t)]$. Further evaluation of $\mathcal{J}$ requires us to manipulate the statistics of the measured optical quadrature $\hat{y}(t)$ and the oscillator motion $\hat{\boldsymbol{x}}_{0}$. We apply the tools introduced in Ref. [26] (i) to simplify the statistics of $\hat{y}(t)$, while maintaining its full information by causally whitening it into $\hat{z}(t)$ such that $\left\langle\hat{z}(t) \hat{z}\left(t^{\prime}\right)\right\rangle=\delta\left(t-t^{\prime}\right)$, and (ii) to separate $\hat{x}_{0}$ into a quantum part $\hat{\boldsymbol{R}}$ and a classical part which can be inferred from $\hat{z}$ by using the optimal Wiener filter $\boldsymbol{K}-\hat{\boldsymbol{x}}_{0} \equiv \hat{\boldsymbol{R}}+$ $\int_{-\infty}^{0} d t \boldsymbol{K}(-t) \hat{z}(t)$ - such that $\hat{\boldsymbol{R}}$ is not correlated with $\hat{z}(t)$, namely $\langle 0|\hat{\boldsymbol{R}} \hat{z}(t)| 0\rangle=0$. With these tools, the path integral can be completed, and it gives

$$
\mathcal{J}=\frac{1}{w(\boldsymbol{y})} \int d^{2} \zeta e^{-\left[\boldsymbol{\alpha} \mathbb{V}_{c} \boldsymbol{\alpha}^{\prime}+\left\|z-2 \zeta \boldsymbol{L}^{\prime}\right\|^{2}\right] / 2+i \boldsymbol{\alpha} x_{\zeta}^{\prime}} P(\zeta) .
$$

Here, $\mathbb{V}_{c} \equiv\left\langle 0\left|\hat{\boldsymbol{R}}^{\prime} \hat{\boldsymbol{R}}\right| 0\right\rangle$ with $\hat{\boldsymbol{R}}=\left(\hat{\boldsymbol{R}}_{x}, \hat{\boldsymbol{R}}_{p}\right)$; the function norm: $\|g\|^{2} \equiv \int_{-\infty}^{0} g(t) g^{*}(t) d t ; \boldsymbol{x}_{\zeta} \equiv \boldsymbol{x}_{c}+\zeta^{*} \boldsymbol{\gamma}+\zeta \boldsymbol{\gamma}^{*}$; $\boldsymbol{x}_{c} \equiv\left(x_{c}, p_{c}\right)=\int_{-\infty}^{0} d t \boldsymbol{K}(-t) z(t)$ with $z(t)$ the measured results of $\hat{z}(t)$, and $\boldsymbol{K}=\left(K_{x}, K_{p}\right) ; \boldsymbol{\gamma} \equiv[\hat{\Gamma}, \hat{\boldsymbol{R}}]$ and $\boldsymbol{L} \equiv$ $(\Re[L], \Im[L])$ with $L(t) \equiv[\hat{\Gamma}, \hat{z}(t)]$. These two parameters $\boldsymbol{\gamma}$ and $\boldsymbol{L}$ characterize the contribution of the photon $\hat{\Gamma}$ to both the oscillator motion $\hat{\boldsymbol{R}}$ and the output field $\hat{z}$, and they determine the efficiency of the state transfer.

Finally, the Wigner function for the quantum state of the oscillator reads (the normalization factor is ignored)

$$
W=\int d^{2} \zeta e^{-\left[\left(\boldsymbol{x}-\boldsymbol{x}_{\zeta}\right) \mathbb{V}_{c}^{-1}\left(\boldsymbol{x}-\boldsymbol{x}_{\zeta}\right)^{\prime}+\left\|z-2 \zeta \boldsymbol{L}^{\prime}\right\|^{2}\right] / 2} P(\boldsymbol{\zeta}) .
$$

This formula directly relates the optical state to the resulting state of the mechanical oscillator. Since only linearity, instead of specific Hamiltonian, is assumed in deriving this formula, it is valid for general linear quantum dynamics. For optomechanical systems, $\boldsymbol{\gamma}, \mathbb{V}_{c}, \boldsymbol{K}$, and $L$ can be obtained from the standard input-output relations in Refs. [27-29] by using the formalism in Ref. [26]. The 

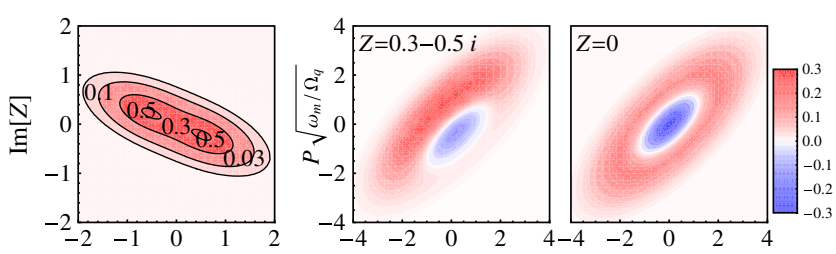

$\operatorname{Re}[Z]$
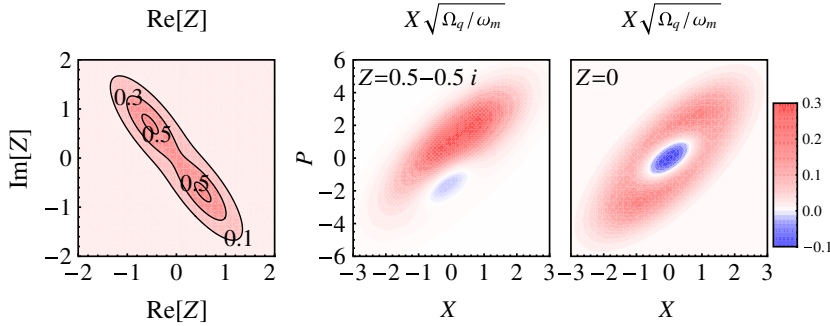

FIG. 3 (color online). Distributions of measurement results (left panels) and the corresponding Wigner function of the oscillator, given the most probable measurement result (middle panels), and less probable result but with a significant nonGaussianity (right panels).

state transfer efficiency can be measured quantitatively by the fidelity defined as $F \equiv \operatorname{Tr}\left[\hat{\rho}_{m}^{c} \hat{\rho}_{o}\right][30,31]$.

Single-photon case.-As an example, we consider the simplest case where the optical field is in a single-photon state with $\hat{\rho}_{o}=|1\rangle\langle 1|$, and $P(\zeta)=e^{|\zeta|^{2}} \partial^{2} \delta^{(2)}(\zeta) / \partial \zeta \partial \zeta^{*}$. From Eq. (16), the normalized Wigner function reads

$$
W=\frac{1-\boldsymbol{\gamma} \mathbb{\bigvee}_{c}^{-1} \boldsymbol{\gamma}^{\dagger}-\|L\|^{2}+\left|\boldsymbol{\gamma} \mathbb{\bigvee}_{c}^{-1} \delta \boldsymbol{x}^{\prime}+Z\right|^{2}}{2 \pi \sqrt{\operatorname{det} \mathbb{V}_{c}}\left(1-\|L\|^{2}+|Z|^{2}\right)} e^{-\delta \boldsymbol{x} \mathbb{V}_{c}^{-1} \delta \boldsymbol{x}^{\prime} / 2,}
$$

where $\delta \boldsymbol{x} \equiv \boldsymbol{x}-\boldsymbol{x}_{c}$, and $Z \equiv \int_{-\infty}^{0} d t z(t) L(t)$. Since the measurement results $z(t)$ only appear in the above Wigner function in terms of an integral, i.e., $Z$, the conditional process is easy to study. The random vector $Z=$ $(\Re[Z], \Im[Z])$ follows a two-dimensional distribution,

$$
w[\boldsymbol{Z}]=\frac{1-\|L\|^{2}+\boldsymbol{Z} \boldsymbol{Z}^{\prime}}{2 \pi \sqrt{\operatorname{det} \mathbb{V}_{L}}} e^{-\boldsymbol{Z} \mathbb{V}_{L}^{-1} \mathbf{Z}^{\prime} / 2},
$$

with $\mathbb{V}_{L} \equiv \int_{-\infty}^{0} d t \boldsymbol{L}^{\prime} \boldsymbol{L}$.

With Eq. (17), we can justify the previous order-ofmagnitude estimate by using the specifications in Table I. As an example, we assume a photon profile of $f(t)=$ $\sqrt{2 \gamma_{f}} e^{\left(\gamma_{f}+i \omega_{f}\right) t}$. The resulting Wigner functions of the mechanical oscillator are shown in Fig. 3: the upper panels show the case of an advanced gravitational-wave detector with $\omega_{f} / 2 \pi=\gamma_{f} / 2 \pi=70 \mathrm{~Hz}, \Omega_{q} \equiv \sqrt{\alpha^{2} / \hbar m}$, and the state transfer fidelity $F=0.58(Z=0.3-0.5 i)$ and $F=$ $0.95(Z=0)$; the lower panels show the case of a smallscale device, with $\omega_{f} / \omega_{m}=0.1, \gamma_{f} / \omega_{m}=0.3$, and the corresponding $F=0.34(Z=0.5-0.5 i)$ and $F=0.56$ $(Z=0)$. In both cases, the Wigner functions have negative regions-a unique quantum feature. The prepared nonGaussian states can be probed with the quantum tomography protocol proposed in Ref. [32], which allows us to reconstruct the quantum state with sub-Heisenberg accuracy, and to reveal these nonclassical negative regions. We can, therefore, prepare and probe non-Gaussian states of the mechanical oscillator.

We thank our colleagues at Caltech Theoretical Astrophysics group and LIGO MQM group for fruitful discussions. We thank R. Schnabel and C. Zhao for useful comments. F. K.'s research has been supported by NSF and Caltech Grant No. PHY-0651036. S. D., H. M.-E., H. Y., and Y.C. are supported by the Alexander von Humboldt Foundation's Sofja Kovalevskaja Programme, NSF Grants No. PHY-0653653 and No. PHY-0601459, as well as the David and Barbara Groce startup fund at Caltech. H. M. is supported by the Australian Research Council and Department of Education.

[1] Review articles by T. J. Kippenberg and K. J. Vahala, Science 321, 1172 (2008); F. Marquardt and S.M. Girvin, Physics 2, 40 (2009).

[2] V.B. Braginsky and F. Khalili, Quantum Measurement (Cambridge University Press, Cambridge, UK, 1992).

[3] A. J. Leggett, Prog. Theor. Phys. Suppl. 69, 80 (1980).

[4] J.S. Bell, Speakable and Unspeakable in Quantum Mechanics (Cambridge University Press, Cambridge, 1987).

[5] S. L. Braunstein and P. van Loock, Rev. Mod. Phys. 77, 513 (2005).

[6] A. D. O'Connell et al., Nature (London) 464, 697 (2010).

[7] A. A. Clerk and D.W. Utami, Phys. Rev. A 75, 042302 (2007).

[8] K. Jacobs et al., Phys. Rev. Lett. 98, 147201 (2007); Phys. Rev. Lett. 99, 117203 (2007).

[9] I. Martin and W. H. Zurek, Phys. Rev. Lett. 98, 120401 (2007).

[10] J. Thompson et al., Nature (London) 452, 72 (2008).

[11] H. Miao et al., Phys. Rev. Lett. 103, 100402 (2009).

[12] D. Kleckner et al., New J. Phys. 10, 095020 (2008).

[13] http://www.ligo.caltech.edu/advLIGO.

[14] C. K. Law and H. J. Kimble, J. Mod. Opt. 44, 2067 (1997).

[15] J. Mckeever et al., Science 303, 1992 (2004).

[16] M. Keller et al., Nature (London) 431, 1075 (2004).

[17] J. Zhang et al., Phys. Rev. A 68, 013808 (2003).

[18] K. Jahne et al., Phys. Rev. A 79, 063819 (2009).

[19] S. Mancini et al., Phys. Rev. Lett. 90, 137901 (2003).

[20] O. Romero-Isart et al., New J. Phys. 12, 033015 (2010).

[21] A. Hopkins et al., Phys. Rev. B 68, 235328 (2003).

[22] C. Gardiner and P. Zoller, Quantum Noise (SpringerVerlag, Berlin, 2004), 3rd ed.

[23] G. Milburn, Quantum Semiclass. Opt. 8, 269 (1996).

[24] A. Doherty et al., Phys. Rev. A 60, 2380 (1999).

[25] A. C. Doherty and K. Jacobs, Phys. Rev. A 60, 2700 (1999).

[26] H. Müller-Ebhardt et al., Phys. Rev. A 80, 043802 (2009).

[27] F. Marquardt et al., Phys. Rev. Lett. 99, 093902 (2007).

[28] I. Wilson-Rae et al., Phys. Rev. Lett. 99, 093901 (2007).

[29] C. Genes et al., Phys. Rev. A 77, 033804 (2008).

[30] B. Julsgaard et al., Nature (London) 432, 482 (2004).

[31] P. Marek and R. Filip, Phys. Rev. A 81, 042325 (2010).

[32] H. Miao et al., Phys. Rev. A 81, 012114 (2010). 